\title{
Friction Experiments for Dynamical Coefficient Measurement
}

\author{
J. J. Arnoux, G. Sutter, G. List, and A. Molinari \\ Laboratoire de Physique et Mécanique des Matériaux, FRE CNRS 3236, Ile du Saulcy, 57045 Metz Cedex 01, France \\ Correspondence should be addressed to J. J. Arnoux, jjarnoux@univ-metz.fr
}

Received 30 November 2010; Revised 1 February 2011; Accepted 10 May 2011

Academic Editor: Dae Eun Kim

Copyright (C) 2011 J. J. Arnoux et al. This is an open access article distributed under the Creative Commons Attribution License, which permits unrestricted use, distribution, and reproduction in any medium, provided the original work is properly cited.

\begin{abstract}
An experimental study, including three experimental devices, is presented in order to investigate dry friction phenomena in a wide range of sliding speeds for the steel on steel contact. A ballistic setup, with an air gun launch, allows to estimate the friction coefficient between $20 \mathrm{~m} / \mathrm{s}$ and $80 \mathrm{~m} / \mathrm{s}$. Tests are completed by an adaptation of the sensor on a hydraulic tensile machine $(0.01 \mathrm{~m} / \mathrm{s}$ to $3 \mathrm{~m} / \mathrm{s}$ ) and a pin-on-disk tribometer mounted on a CNC lathe (1 to $30 \mathrm{~m} / \mathrm{s})$. The interactions at the asperity scale are characterized by a white light interferometer surface analysis.
\end{abstract}

\section{Introduction}

Friction is a crucial parameter in many engineering applications when contact surfaces are subjected to heat and wear. The interaction between two solids is often modelled through the Coulomb friction law, connecting the normal stress with the tangential stress $\left(\tau_{f}=\mu \sigma_{n}\right)$. In many models applied to metal forming, the friction coefficient $\mu$ is represented by a constant value which depends on the nature of materials and surface roughness. However, when the sliding speed and the contact pressure tend toward extreme conditions, a better evaluation of the friction coefficient is necessary to correctly represent the physical phenomena. For example, in high speed machining, modelling the friction coefficient as a function of sliding speed and contact pressure can account for the evolution of cutting forces at different cutting conditions [1]. Moreover, the combination of high pressures and high sliding speeds increases the difficulty of experimental determination of this coefficient [1-6]. For the tests reliability, the local conditions of high pressure, high speed and surface characteristics of the studied processes must be reproduced. These conditions must be achieved with a simple geometry for which the measurement of contact parameters is easily. Besides, the combination of different apparatus is often necessary to characterize the friction coefficient in a wide range of contact conditions In this context, three complementary devices for measuring dynamic friction are developed with contact pressures greater than $250 \mathrm{MPa}$ : (1) a device of pin-on-disc mounted on CNC lathe, (2) a friction device installed on a rapid hydraulic machine, and (3) an experiment performed by using a ballistic device (gas launcher). The superposition of speed ranges obtained with the different devices also allows a validation of results. The pair steel on steel (C22) is selected to study the interaction on the roughness scale characterized by junctions and microwelds. The evolution of friction coefficient is determined in the range of sliding velocity from $0.01 \mathrm{~m} / \mathrm{s}$ to $80 \mathrm{~m} / \mathrm{s}$.

\section{Experimental Devices}

An original sensor for measuring forces generated during friction was developed to combine the high sliding speed and high contact pressure. The specific load sensor (Figure 1) is integrated on a ballistic bench (Figure 2) able to reach sliding speeds above $120 \mathrm{~m} / \mathrm{s}$. Two additional devices are used to expand the range of explored sliding speeds and to validate the measurements made by different acquisition channels. These devices are firstly a hydraulic dynamic testing machine (Figure 3(a)) capable of receiving the sensor and another one is a more conventional type of pin-on-disk (Figure 3(b)). The ballistic device presented in Figure 2 is composed of two coaxial tubes: the first one is the launch tube and the second the receiving tube leading to a shock absorber. This equipment was originally developed to study extreme cutting conditions [7] in a perfectly orthogonal cutting configuration without drawback induced by industrial machines. The quasi-instantaneous expansion of a compressed air stored in 


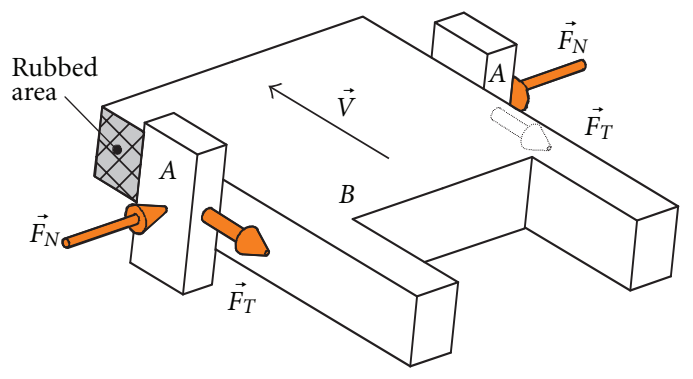

(a)

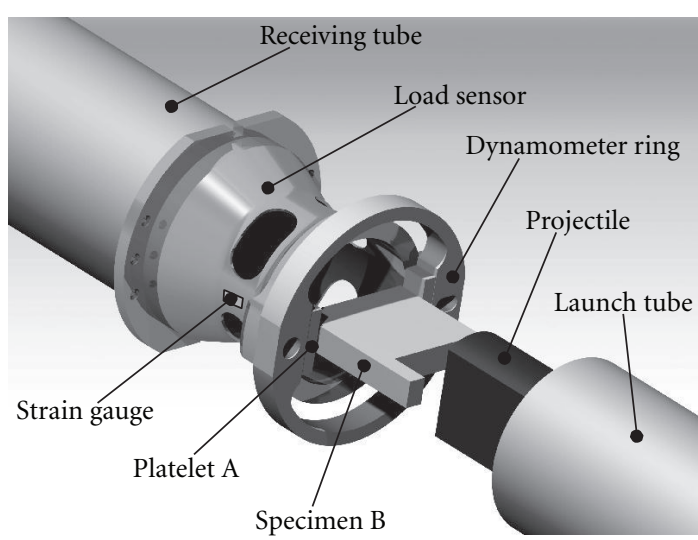

(b)

Figure 1: Details of the dynamical tribometer.

an accumulator propels a projectile at high speed into the launching tube. A sufficient length of this tube, combined with an adjusted projectile mass (different geometries and different materials in order to have a suitable kinetic energy) based on testing parameters, provides almost constant speed (less than $4 \%$ variation) to the projectile during the sliding process. A set of three sensors record accurately the velocity and acceleration of the projectile.

The load sensor (cf. Figure 1), attached to the receiving tube, supports specimen $A$ and imposes a normal pressure via a dynamometer ring. The translation motion of specimen $B$ is caused by an inelastic collision with the projectile. Specimen $B$ is received in a shock absorber for postmortem analysis of the rubbed surfaces. The load sensor was designed to be easily used with another propeller as a more conventional high speed servohydraulic machine. This assembly, shown schematically in Figure 3(a), allows to explore the range of low friction velocities $(0.01 \mathrm{~m} / \mathrm{s}<V<3 \mathrm{~m} / \mathrm{s})$. The hydraulic jack of the tensile machine replaces the projectile and ensures the displacement of specimen $B$ pinched by the two platelets $A$. The measurement of forces generated by the friction will be twofold, firstly from the load cell of the machine and secondly by the sensor.

The third friction device of pin-on-disk type is mounted on a CNC lathe (see Figure 3(b)). The mandrel provides the rotation of the specimen (disc) while the platelet (pin) is fixed to the tool holding fixture. Force measurement is performed by a Kistler dynamometer, positioned under the support of the pin. All the tests presented in this study are conducted under dry friction conditions for a middle hard steel C22 $\left(R_{e}=340 \mathrm{MPa}\right)$ on itself. The friction surfaces are obtained by grinding in a direction similar to rubbing movement $\left(R_{a}=0.9 \mu \mathrm{m}\right)$. It is important to note that the friction tests are carried out with a single pass between two new surfaces (not running-in phase). This approach helps to understand the fundamental phenomena present during the single interaction of two surfaces. Samples of rubbing surfaces are analysed with a white light interferometer. These observations allow us to follow the evolution of the

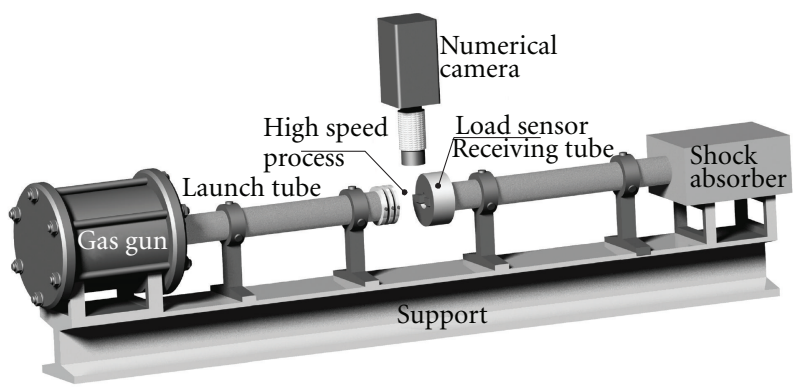

FIgURE 2: Ballistic setup dedicated to the dynamical friction study.

roughness and also to observe the particles snatched by the microjunctions.

\section{Characterization of the Frictional Force}

The friction coefficient $\mu$ is defined through the forces measurement:

$$
\mu=\frac{F_{T}}{F_{N}},
$$

where $F_{T}$ is the frictional or tangential force and $F_{N}$ is the normal force. During the three types of tests, the sliding speed $V$ and the normal force are imposed. The apparent pressure normal to the contact surfaces is defined by

$$
P_{a}=\frac{F_{N}}{S_{a}}
$$

where $S_{a}$ is the apparent contact surface. The typical signal recorded during the tests on the ballistic device is presented in Figure 4. The signals of the two strain gages are combined and averaged to correct any misalignment at impact which could cause a discontinuity of friction. To insure the guidance of specimen $B$ by the projectile through the 


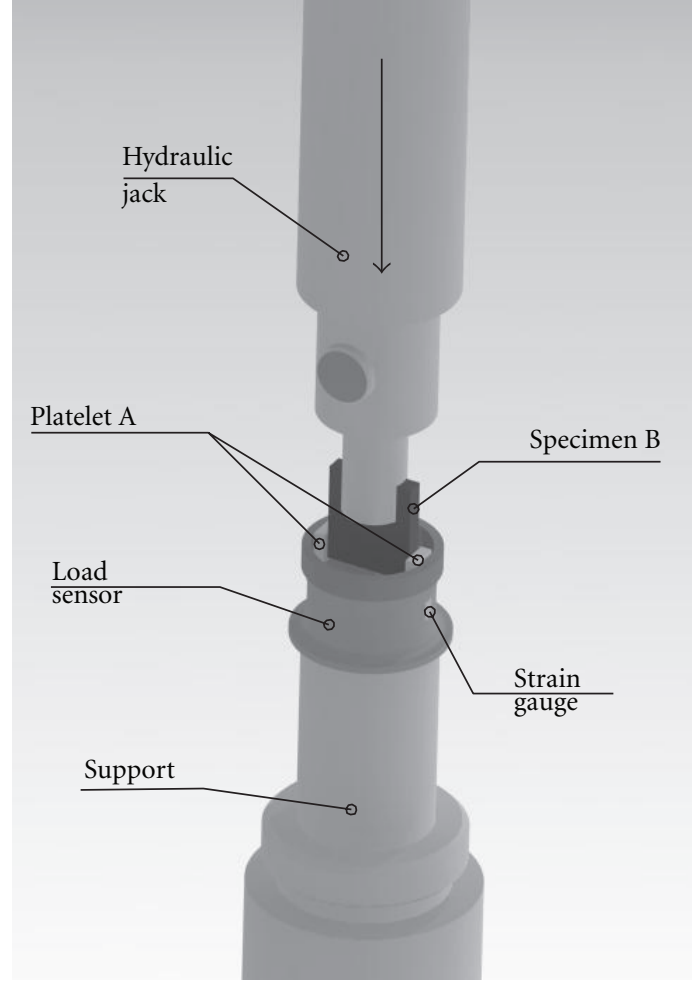

(a)

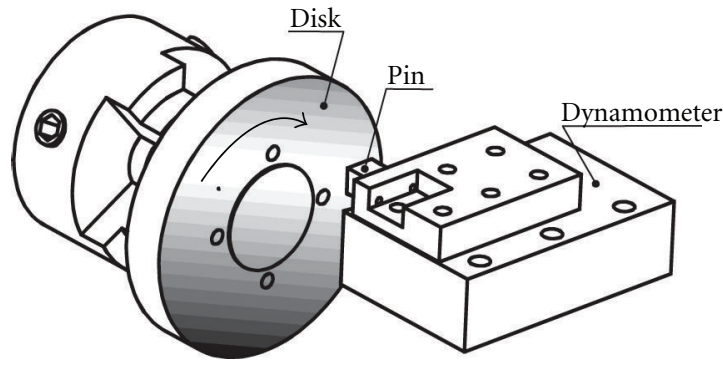

(b)

Figure 3: (a) Load sensor adapted for a servohydraulic machine. (b) Pin-on-disk tribometer mounted on a CNC lathe.

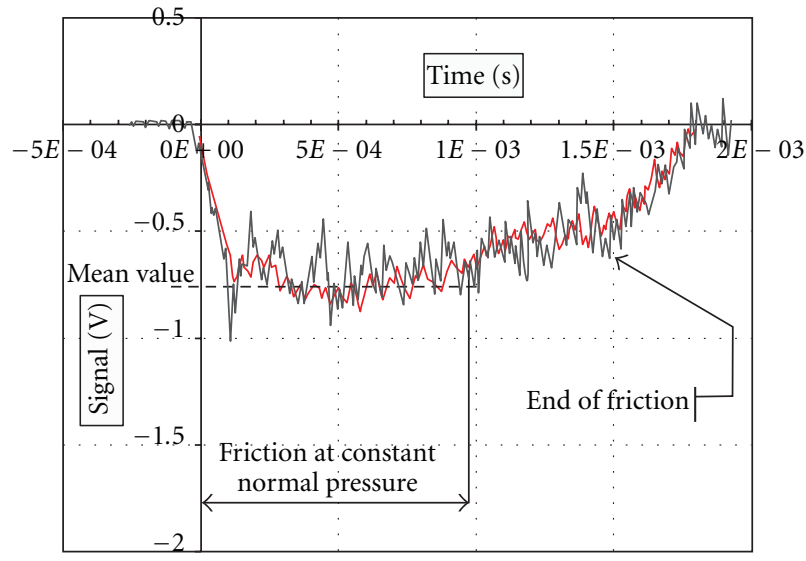

- Average signal

- Filtered signal

Figure 4: Typical signal obtained on the ballistic device $(V=35 \mathrm{~m} / \mathrm{s}$, $\left.P_{a}=40 \mathrm{MPa}\right)$.

reception tube, a particular geometry (illustrated in Figure 1) is defined. Inconstant stiffness is then observed causing a pressure decrease at the end of the test. Only the portion of the signal corresponding to the zone of constant pressure is taken into account to determine the frictional force. With the pin-on-disk tribometer, it is furthermore possible to monitor

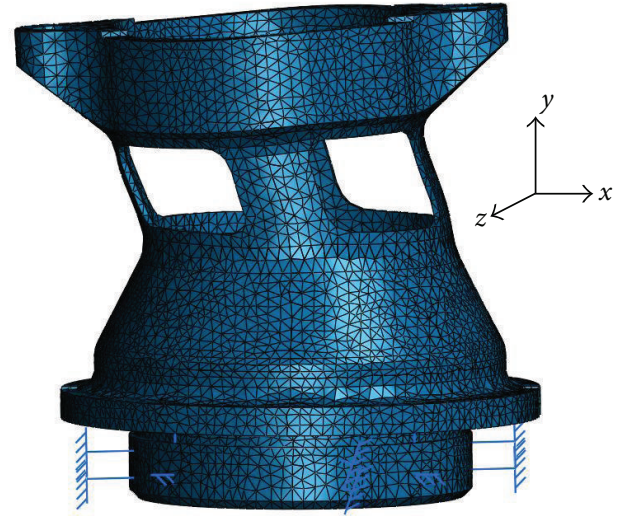

FIGURE 5: Principal vibration eigenmodes of the sensor at $2169 \mathrm{~Hz}$ (flexure mode around the $z$-axis).

and record the normal force applied and the force due to friction during the test. Unlike other presented devices, which use the dynamometer ring, the application of the pressing force and the unloading are not instantaneous.

Moreover, the dynamical effects due to the elevated speed require a special analysis of the recorded signal. Several 


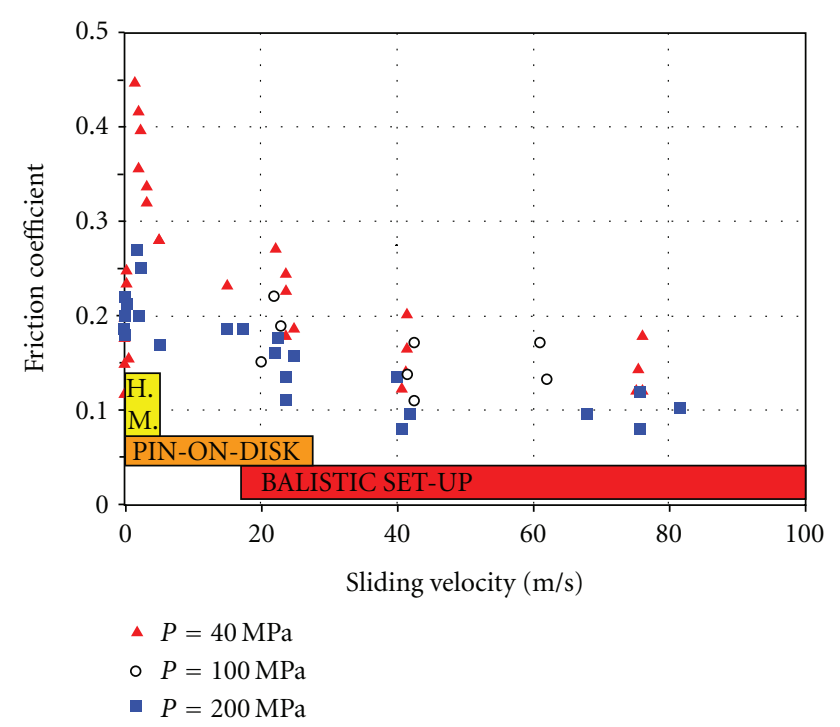

Figure 6: Evolution of the friction coefficient for a steel on steel contact.

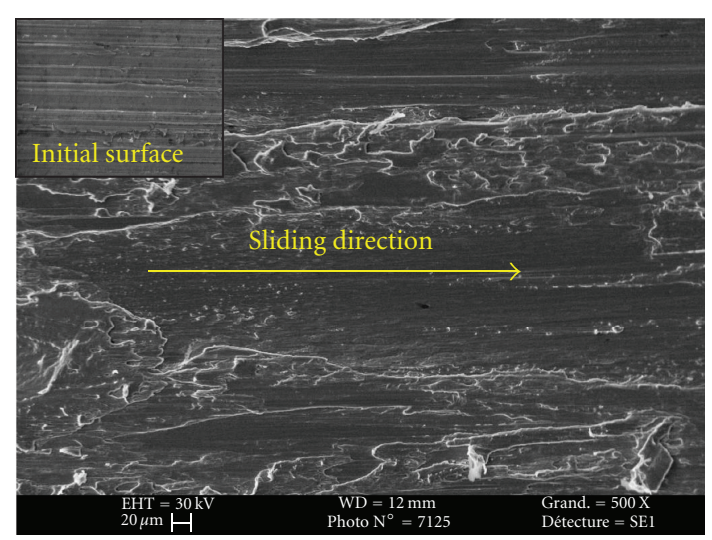

FIGURE 7: SEM photographs (magnitude $\times 500$ ) after friction, $V=$ $20 \mathrm{~m} / \mathrm{s}, \mathrm{P}_{\mathrm{a}}=40 \mathrm{MPa}$.

vibration modes are presented on the signal. A treatment is proposed in order to differentiate the main eigenmodes of the load sensor and the frequencies induced the by frictional phenomenon. The different eigenmodes are estimated by vibration analysis using a finite element modelling (see Figure 5). Effective treatment of the signal from the gauges (to remove the eigenmode frequencies) becomes possible (bold line in Figure 4). The mean value of the friction coefficient is deduced from the filtered signal (horizontal broken line in Figure 4).

\section{Results and Comments}

Figure 6 presents the friction coefficient as a function of the sliding speed for different contact pressures. Thanks to the complementarity of the three devices, the evolution of the friction coefficient is determined for speeds ranging from
$0.01 \mathrm{~m} / \mathrm{s}$ to $80 \mathrm{~m} / \mathrm{s}$. The friction coefficient trends to decrease with the sliding speed, as also shown in previous work with similar development $[5,6]$.

The fall of the coefficient $\mu$ is attributed to the effect of temperature which increases with speed. From $30 \mathrm{~m} / \mathrm{s}$, a smaller variation of friction is nevertheless observed. At higher velocity, the effect of temperature is in competition with the effect of viscous behaviour (increase of the stress with strain rate). Pressure and sliding velocity directly affect the conditions of contact of the asperities. The deformation process generated by the friction leads to the reconstruction of connectivity between the asperities of the two solids. More or less strong junctions are created, and the condition of large sliding speed accelerates the mechanism of shear and alteration of the asperities; see Figure 7.

To explain the evolution of the friction coefficient, all thermomechanical phenomena involved during the shearing of asperities must be taken into account $[5,6,8]$. The normal force and tangential force can be evaluated by the following equations:

$$
\begin{gathered}
F_{T}=f \tau S_{r}, \\
F_{N}=\sigma S_{r},
\end{gathered}
$$

where $S_{r}$ is the real contact area, $\tau$ the shear stress of asperities, and $\sigma$ the normal stress at the junctions. The factor $f(0 \leq f \leq 1)$ represents the fraction of the real contact area which actually contributes to shear. The real surface $S_{r}$ is related to the apparent surface $S_{a}$ by the coefficient $\alpha(0 \leq \alpha \leq 1)$

$$
S_{r}=\alpha S_{a} .
$$

The determination of $f$ and $\alpha$ is necessary to describe and model the evolution of the friction coefficient. For example, $\alpha$ increases with the normal force by increasing the number of junctions between the asperities. Nonetheless, the values of these parameters cannot be easily determined through experimental observations. Surface analysis can be achieved using white light microscopy, by reconstructing the 3D surface profiles. Illustrations in Figure 8 show the evolution of the sliding surface of platelet A (fixed part) after a friction test on the ballistic device at $20 \mathrm{~m} / \mathrm{s}$ and with an apparent pressure of $40 \mathrm{MPa}$.

The rupture of the junctions leads to a mass transfer between the two parts in contact up to ten microns in height, as it can be observed in Figure 8. The surface roughness $R_{a}$ is severely deteriorated shifting from $0.9 \mu \mathrm{m}$ to $3.0 \mu \mathrm{m}$. Increasing the speed and pressure modifies the contact conditions, and a change of the surfaces takes place by clipping the peaks of asperities. Figure 9 shows the surface of a specimen before and after rubbing with $V=60 \mathrm{~m} / \mathrm{s}$ and $P_{a}=100 \mathrm{MPa}$. A higher pressure significantly increases the real contact area $S_{r}$. However, at the scale of asperities, the volume of the sheared material is considerably reduced (on only few micrometers in height). Contrary to the test described above (lower pressure and speed), the surface roughness is somewhat improved because the value of $R_{a}$ decreases from $0.9 \mu \mathrm{m}$ to $0.82 \mu \mathrm{m}$. In addition, although the number of contacts has increased, the 


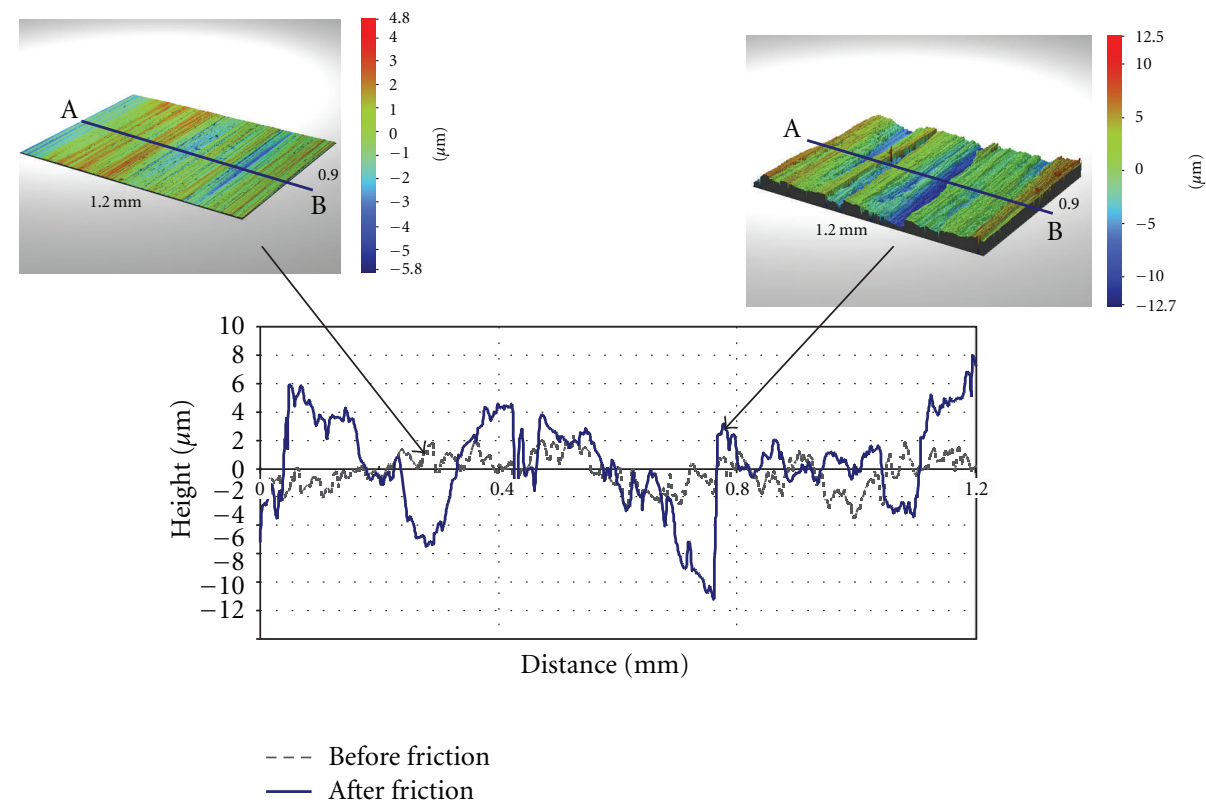

FIGURE 8: Surface topographies for conditions $V=20 \mathrm{~m} / \mathrm{s}$ and $P_{a}=40 \mathrm{MPa}$ and superposition of the 2D profiles (along the line $\mathrm{AB}$ ).

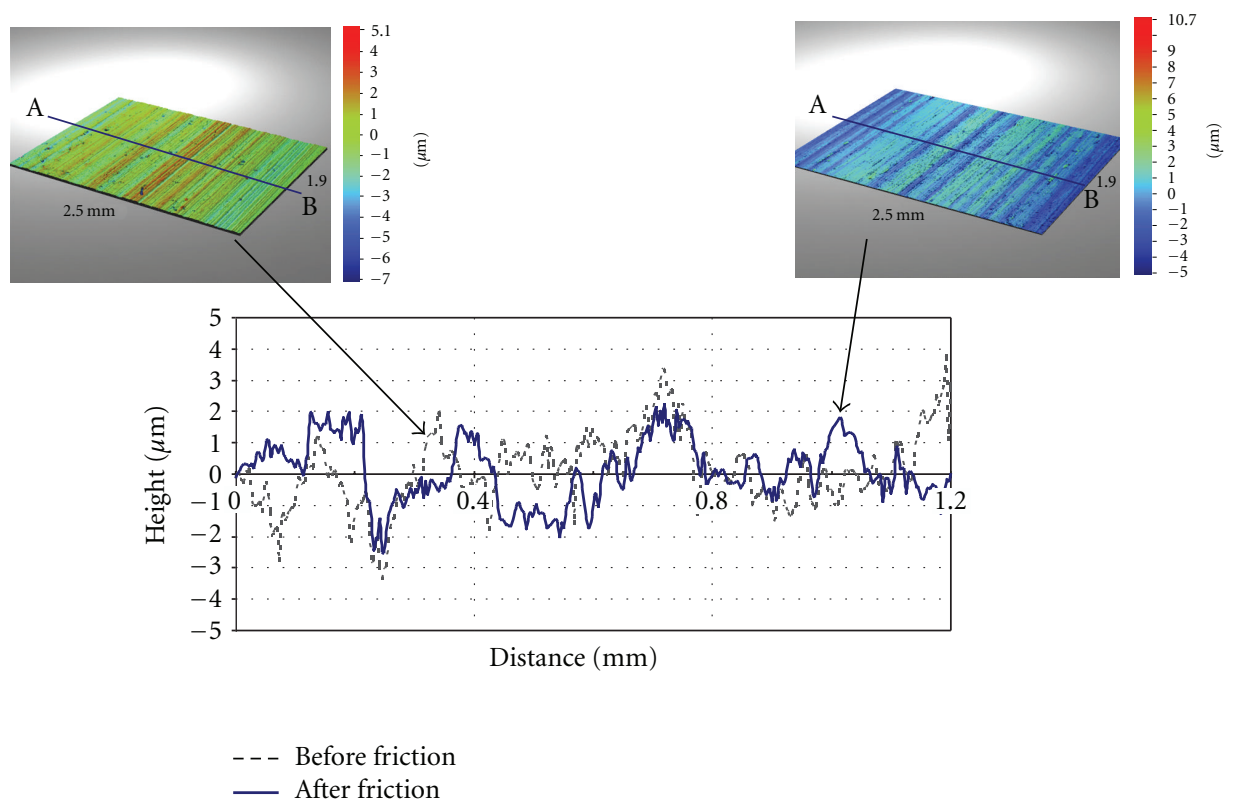

Figure 9: Surface topographies for conditions $V=60 \mathrm{~m} / \mathrm{s}$ and $P_{a}=100 \mathrm{MPa}$ and superposition of the 2D profiles (along the line $\mathrm{AB}$ ).

observation suggests that only a small proportion of the junctions were effectively sheared. In this case, a lower value of the parameter $f$ must be considered. The evolution of the surface topography can be explained considering the change of plastic flow regime. Indeed, it was observed that high sliding velocity and great pressure can generate conditions leading to interfacial temperatures approaching the near-melt and fully melt temperature regime [9-11]. Local melting occurs at the asperity junctions contributing to the development of molten metal films at the tribo-pair interface.

The knowledge of the parameters $\alpha$ and $f$, which depend on test conditions, is however not sufficient to explain the global evolution of the friction coefficient. The material behaviour and temperature rise must also be taken into 
account. The friction coefficient can be written as follows where the pressure $P_{a}$ is assumed to be constant during a test:

$$
\mu=\frac{f \tau \alpha S_{a}}{F_{N}}=\frac{f \alpha \tau}{P_{a}} .
$$

The shear stress $\tau$ is related to material thermo-viscoplastic behaviour and can be expressed as a function of the shear strain $\gamma$, the shear strain rate $\dot{\gamma}$, and the temperature $T$ in the deformed asperity:

$$
\tau=\tau(\gamma, \dot{\gamma}, T) .
$$

The strain rate $\dot{\gamma}$ can be estimated by the following equation:

$$
\dot{\gamma}=\frac{V}{h},
$$

where $h$ is the height over which the asperities shearing occurs. According to the observations, the value of height is of order of a few $\mu \mathrm{m}$ leading to a very high strain rate above $10^{6} \mathrm{~s}^{-1}$. While the strain rate tends to increase the stress, the temperature tends to reduce it by thermal softening process. A good description of the temperature can be given by the measurement of the flash temperature $T_{\max }$ which is reached at the contact. Previous results [9] have shown that the flash temperature in the same conditions of contact is over $1000^{\circ} \mathrm{C}$. Finally, if all parameters appearing in (5) were known with accuracy, it would be possible to predict the value of the coefficient $\mu$ and its asymptotic evolution with speed and pressure.

\section{Conclusion}

The analysis of dynamic friction requires knowledge of precise experimental data describing the thermomechanical phenomena which occur in contact mechanisms. In this context, a set of three complementary measurement friction devices were developed. The study concerns the dry friction of steel on steel, but the design of these devices enables a simple adaptation to the study of other pairs of materials. The evolution of the friction coefficient could be observed in a very wide range of sliding speeds and for different pressure levels. The convergence of results on these devices improves the reliability of experimental data. At high speeds, the effect of temperature seems to be predominant. It causes, due to a "thermal softening," a drop in the friction coefficient. However, depending on the applied pressure, the friction coefficient may continue to fall (extreme pressure) or slightly increase (lower pressure). To better understand these variations, the contact parameters such as the actual surface, the sheared volume of material, or the contact temperature at the asperities must be quantified. The combination of high speed and high pressure appears to reduce the surface roughness of the rubbing surfaces due to localized plastic flow and melting. This could explain the asymptotic trend of the coefficient in extreme conditions.

\section{References}

[1] G. Sutter and A. Molinari, "Analysis of the cutting force components and friction in high speed machining," ASME
Journal of Manufacturing Science and Engineering, vol. 127, no. 2, pp. 245-250, 2005.

[2] G. Sutter, S. Philippon, and A. Molinari, "An experimental investigation of dry friction for a large range of sliding velocities," Matériaux et Techniques, pp. 33-37, 2004.

[3] S. Rajagopalan and V. Prakash, "A modified torsional Kolsky bar for investigating dynamic friction," Experimental Mechanics, vol. 39, no. 4, pp. 295-303, 1999.

[4] H. D. Espinosa, A. Patanella, and M. Fischer, "A novel dynamic friction experiment using a modified Kolsky bar apparatus," Experimental Mechanics, vol. 40, no. 2, pp. 138-153, 2000.

[5] F. P. Bowden and E. H. Freitag, "The friction of solids at very high speeds I. Metal on metal; II. Metal on diamond," Proceedings of the Royal Society of London series A: Mathematical and physical sciences, vol. 248, pp. 350-367, 1958.

[6] S. C. Lim, M. F. Ashby, and J. H. Brunton, "The effects of sliding conditions on the dry friction of metals," Acta Metallurgica, vol. 37, no. 3, pp. 767-772, 1989.

[7] G. Sutter, "Chip geometries during high-speed machining for orthogonal cutting conditions," International Journal of Machine Tools and Manufacture, vol. 45, no. 6, pp. 719-726, 2005.

[8] A. Molinari, Y. Estrin, and S. Mercier, "Dependence of the coefficient of friction on the sliding conditions in the high velocity range," Journal of Tribology, vol. 121, no. 1, pp. 35-41, 1999.

[9] G. Sutter and N. Ranc, "Flash temperature measurement during dry friction process at high sliding speed," Wear, vol. 268, no. 11-12, pp. 1237-1242, 2010.

[10] M. Okada, N. S. Liou, V. Prakash, and K. Miyoshi, “Tribology of high-speed metal-on-metal sliding at near-melt and fullymelt interfacial temperatures," Wear, vol. 249, no. 8, pp. 672686, 2001.

[11] F. Yuan, N. S. Liou, and V. Prakash, "High-speed frictional slip at metal-on-metal interfaces," International Journal of Plasticity, vol. 25, no. 4, pp. 612-634, 2009. 

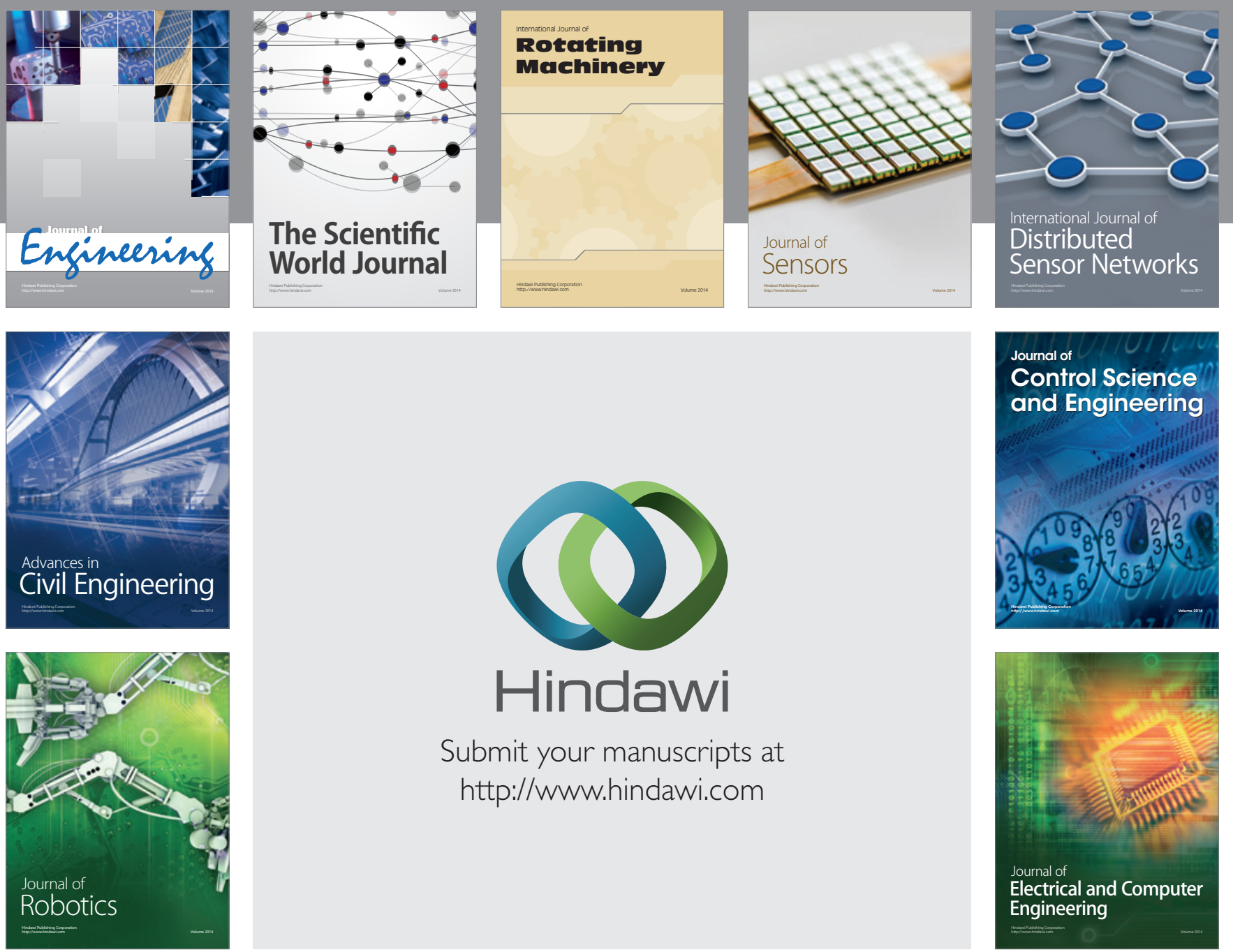

Submit your manuscripts at

http://www.hindawi.com
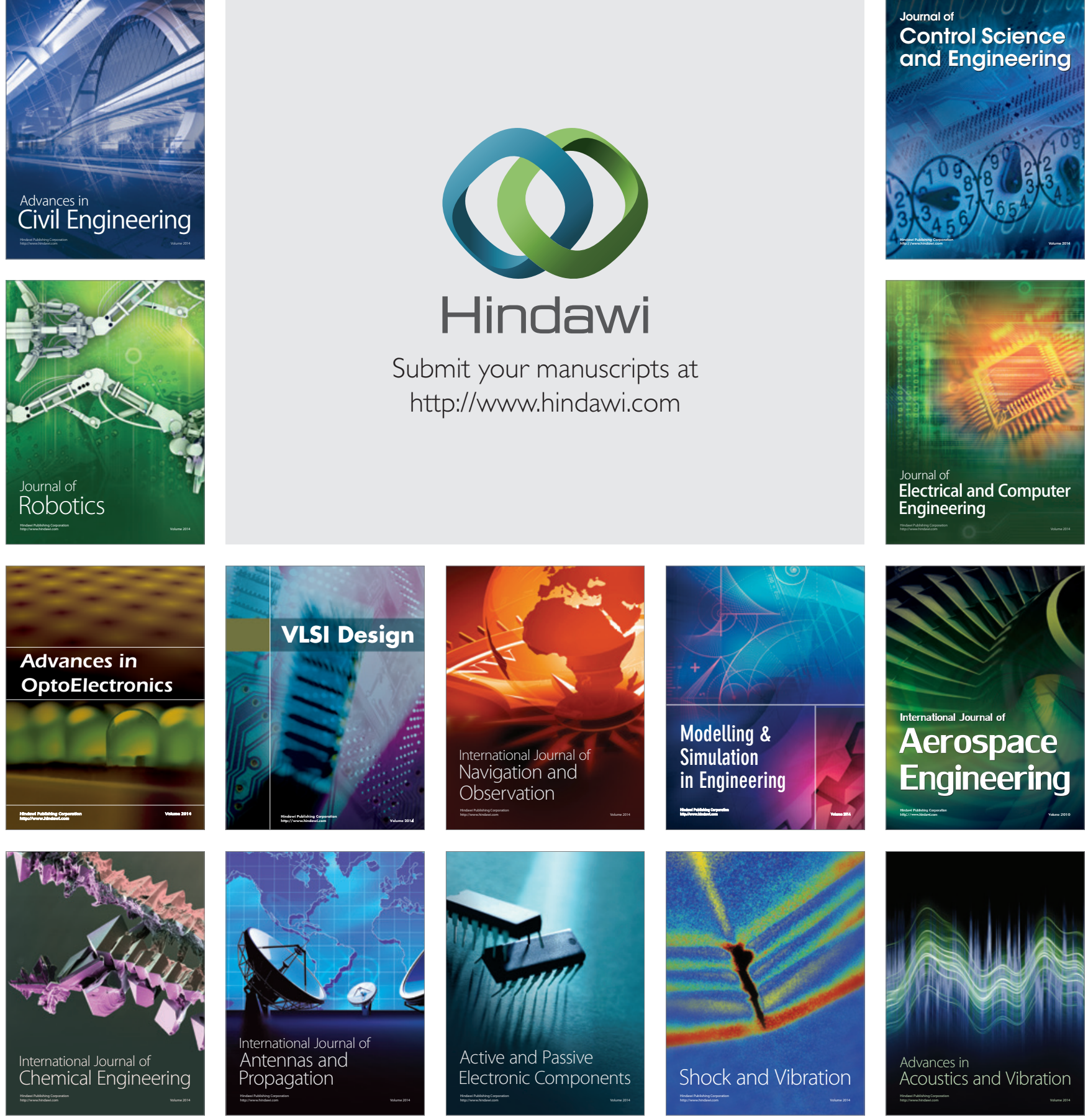